\title{
Evaluation of a personal device in reducing the risk of low back disorders during stooped work
}

\author{
Brent L. Ulrey ${ }^{\mathrm{a},}$ and Fadi A. Fathallah ${ }^{\mathrm{a}^{*}}$ \\ ${ }^{a}$ Occupational Biomechanics Laboratory, Department of Biological and Agricultural Engineering, University of \\ California, One Shields Ave, Davis, CA 95616, USA.
}

\begin{abstract}
Adoption of the stooped posture in the workplace is widespread throughout the world in agriculture, construction, and mining. This type of posture has been established as a risk factor for developing low back disorders (LBDs). The purpose of this study is to evaluate a personal weight transfer device as a possible intervention to reduce the load on the lumbar spine, thereby reducing the risk of developing LBDs. Eighteen healthy subjects performed stooped posture tasks in a laboratory study designed to simulate harvesting of low-growing crops. Results showed that when wearing the device in the static stooped posture, biceps femoris activity was reduced by $17 \%$, lumbar flexion was reduced by $12 \%$, ankle plantar-flexion increased by $5 \%$, and the lumbar erector spinae of those subjects who did not experience flexion-relaxation of the erector spinae was reduced by $26 \%$. Hip and knee flexion were not significantly altered. Therefore, the device may be beneficial for those with existing LBDs, and who use the stooped posture routinely.
\end{abstract}

Keywords: weight transfer device, ergonomic intervention, electromyography

\section{Introduction}

Stooped work, consisting of tasks requiring frequent and/or sustained trunk flexion, is common to jobs in many industries, and is most prevalent in the agriculture, construction, and mining industries. The common link among stooped work jobs in these and other industries is either a low-lying or ground level workspace or a restricted height workspace, which is generally difficult or impossible to modify using traditional ergonomic principles. For instance, in construction, many tasks, such as ground-level reinforcement bar (rebar) construction, require workers to adopt the stooped posture. . In mining, most tasks are performed in restricted height and space environments, requiring the workers to perform their tasks while in the stooped and/or the kneeling posture. On the other hand, in agriculture, stooped work represents a fairly prevalent and unique situation, where workers commonly perform tasks in open fields with little space restrictions, and involving use of equipment or substantial lifting [2]. Because of the difficulty in making ergonomic improvements to stooped work jobs, low back disorders (LBDs) are still highly prevalent in these industries [1].

Recently, several commercial products have been made available, which claim to reduce lower back loading in the stooped posture by moving some of the spinal load to the legs. These products are referred to as weight transfer devices (WTDs). The purpose of this study is to evaluate if a personal weight transfer device is an effective means to reducing the load on the lumbar spine, thereby reducing the risk of developing LBDs.

\section{Methods}

Eighteen healthy subjects (11 male, 7 female) performed stooped work in a laboratory study designed to simulate working tasks in the stooped posture. Average/standard deviation of the participants' age,

*Corresponding author. E-mail: Fathallah@ucdavis.edu 
height and weight were $26 / 8.9$ years, $172.5 / 13.4 \mathrm{~cm}$, and $73 / 9.6 \mathrm{~kg}$, respectively. None of the subjects reported having prior, or current, spinal disorders or back pain.

The BNDR WTD device (Limbic Systems Inc, Ventura, CA) was chosen to be evaluated in this study because of its relative simpler design, and a possible higher potential for acceptance by workers in the field (Figure 1)

Muscle electromyographical activities (EMG), lumbar curvature, and loads in the spine and legs were monitored during the experiment were digitally sampled at $1000 \mathrm{~Hz}$ with Biopac MP150 System hardware and software (version 3.7.3, Biopac Systems, Inc., Santa Barbara, CA). Knee exion and ankle dorsi-exion/plantar-exion angles were sampled at $1000 \mathrm{~Hz}$ using Biometrics electrogoniometers (SG150 and SG110A, respectively), hardware and software (version 2.0, Biometrics Ltd., UK). To measure lumbar and thoracic exion, microelectromechanical systems (MEMS) accelerometers (ADXL203; Ana$\log$ Devices, Norwood, MA) were con_gured as inclinometers $[3,5]$.

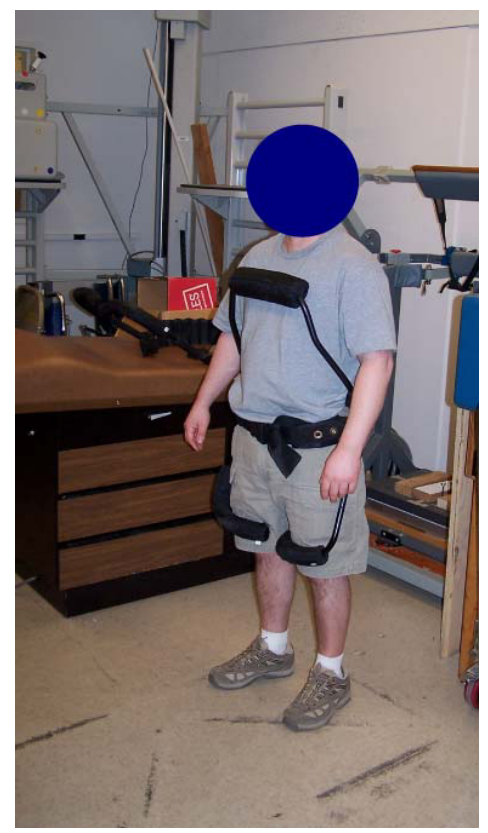

Fig 1 . The BNDR worn by a participant.

The experiment is a 2 X 3 (device X weight) splitplot design. There were two device levels: no device and BNDR. There were three weight levels: no weight, $44.5 \mathrm{~N}$ (10.01b), and 89.0N (20.0lb).
Data processing was performed in a custom Matlab program (version 7.0, The MathWorks, Inc., Natick, MA). The task EMG for each muscle was normalized with the corresponding peak, smoothed EMG in the maximum flexion trials, and is presented as a percentage of this maximum. Data from the knee and ankle goniometers, and the inclinometers, were digitally filtered with a $100 \mathrm{~ms}$ time window. The two filtered channels of each inclinometer were used to calculate its angle with respect to ground. Analysis of variance of the split-plot design was performed on all the dependent variables using the MIXED procedure in SAS (version 9.2, SAS Institute Inc., Cary, $\mathrm{NC)}$.

\section{Results}

Results showed that when wearing the device in the static stooped posture, biceps femoris (BF) activity was reduced by $17 \%$ (Figure 2 ). There was no signicant change in the activities of the lumbar erector spinae (LES), the thoracic erector spinae (TES), the rectus abdominis (RA), or the tibialis anterior (TA) between the device and the no device conditions. Further analysis showed that the lumbar erector spinae of those subjects who did not experience the "flexion-relaxation phenomenon" [4,6-7] of the erector spinae was significantly reduced by $26 \%$. Lumbar flexion was significantly reduced by $12 \%$ and ankle plantar-flexion increased by $5 \%$. Hip and knee flexion were not significantly altered.

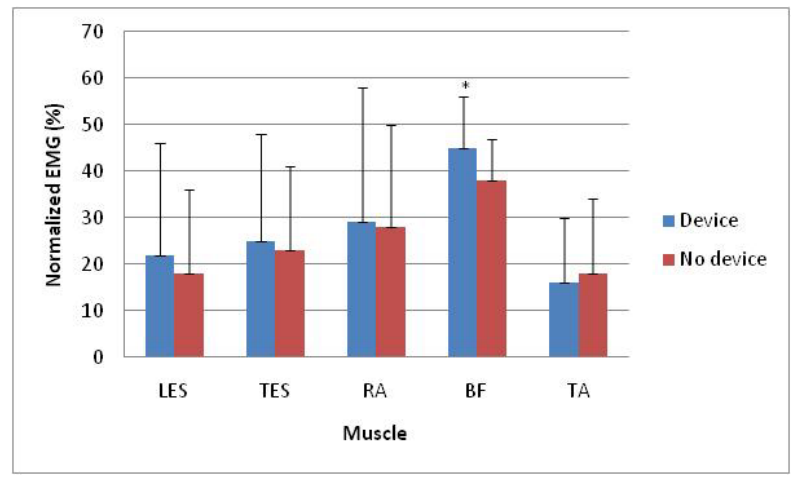

Fig 2. Normalized EMG of the five muscles with and without wearing the device. 


\section{Discussion}

The hypothesis that weight transfer devices significantly reduce back muscle activity during static stooped postures was not supported for all subjects. However, results from this study found a significant reduction in LES activity when wearing the device in those subjects who did not experience flexionrelaxation of the back muscles. With the device, there was a significant reduction in the BF activity observed in the combined dataset. Another important finding is the ability of the device to reduce torso flexion during stoop. The stooped posture is characterized by large trunk flexions. The device prevents flexion of the torso past a certain point, which requires a person to flex their hips to complete a task close to the ground. The point of restriction is dependent on the stiffness of the device. An adjustable stiffness, as well as adjustable or more available sizes, should improve the benefit across the general population.

\section{Acknowledgements}

This work was supported by funds from the Biological and Agricultural Engineering Department, and the Western Center for Agricultural Health and Safety, at the University of California, Davis.

\section{References}

[1] BLS, Occupational Outlook Handbook, 2010-11 Edition, in: D.o. Labor (Ed.), Bureau of Labor Statistics (BLS), Washington, DC, 2011.

[2] F.A. Fathallah, B.J. Miller, J.A. Miles, Low back disorders in agriculture and the role of stooped work: scope, potential interventions, and research needs, J Agric Saf Health 14 (2) (2008) 221-245.

[3] S. Luczak, W. Oleksiuk, M. Bodnicki, Sensing tilt with MEMS accelerometers, IEEE Sensors Journal 6 (6) (2006) 1669-1675.

[4] R.W. McGorry, S.M. Hsiang, F.A. Fathallah, E.A. Clancy, Timing of activation of the erector spinae and hamstrings during a trunk flexion and extension task, Spine 26 (4) (2001) 418-425.

[5] B.J. Miller, F.A. Fathallah, J.A. Miles, Development of an approach for assessing the effects of stooped agricultural work on the lower back spinal tissues, Annual International Meeting of the American Society of Agricultural and Biological Engineers, Portland, OR, 2006.

[6] T. Sihvonen, Flexion relaxation of the hamstring muscles during lumbar-pelvic rhythm, Archives of Physical Medicine and Rehabilitation 78 (5) (1997) 486-490.

[7] T. Sihvonen, J. Partanen, O. Hanninen, S. Soimakallio, Electric behavior of low back muscles during lumbar pelvic rhythm in low back pain patients and healthy controls, Archives of Physical Medicine and Rehabilitation 72 (13) (1991) 1080-1087. 